\title{
Effective Control of Brain Metastases Irrespective of Distance from Isocenter in Single-isocenter Multitarget Stereotactic Radiosurgery
}

\author{
KEN AOKI, YUKIHIRO NAGATANI, KAZUO NOMA, TAKUYA TSUGAWA, NAOAKI KONO, \\ YUKAKO KIDA, NAOKI HARADA, YOSHIHIDE TANOUE and YOSHIYUKI WATANABE \\ Department of Radiology, Shiga University of Medical Science, Otsu, Japan
}

\begin{abstract}
Background/Aim: Few previous studies have evaluated the effectiveness of single-isocenter multitarget (SIMT) stereotactic radiosurgery (SRS) in clinical practice. Patients and Methods: Gross tumor volumes of 113 metastases in 13 patients were measured by contrastenhanced magnetic resonance imaging. Prescribed doses were set at 20-24 Gy. Based on tumor reduction rates (TRRs) measured before and after SIMT SRS, tumor shrinkage effect was categorized into four grades; almost disappeared: $T R R=1$, decreased: $0.3 \leq T R R<1$, stable: $-0.2<T R R<0.3$ and increased: TRR $\leq-0.2$. Tumor shrinkage effects were compared among 3 groups; near group with a distance of $<3.2 \mathrm{~cm}$, middle group with a distance of $\geq 3.2 \mathrm{~cm}$ and $<6.4$ $\mathrm{cm}$, and far group with a distance of $\geq 6.4 \mathrm{~cm}$, categorized by distance from the isocenter. Results: Median survival time was 17 months, with $63.7 \%, 11.5 \%$ and $12.4 \%$ of metastases corresponded to almost disappeared, decreased and stable, respectively. No significant difference was found in the distribution for TRRs among 3 groups. Conclusion: Good local control of multiple brain metastases was demonstrated by SIMT SRS, irrespective of distance from the isocenter.
\end{abstract}

Cancers derived from various organs can metastasize to the brain $(1,2)$. The lungs are the most common site of primary cancer that metastasizes to the brain, which accounts for 40 $52 \%$ of brain metastases (3). Steroid therapy, local excision,

This article is freely accessible online.

Correspondence to: Ken Aoki, Department of Radiology, Shiga University of Medical Science, Seta tsukinowa-cho, Otsu, Shiga 520-2192, Japan. Tel: +81 775440986, +81 775482288, e-mail: kaoki@belle.shiga-med.ac.jp

Key Words: Single-isocenter, stereotactic radiosurgery, dynamic conformal arc, tumor quantification, lung cancer, brain metastasis, contrast-enhanced magnetic resonance imaging. and radiation therapy are often selected as treatments for brain metastases (4). Whole-brain irradiation has been the most common radiation therapy for patients with more than three multiple brain metastases, and its efficacy has been well established (1). However, it has been suggested that cognitive decline may occur after whole-brain irradiation (5, $6)$. Due to recent improvements in systemic therapy with molecular targeting drugs such as epidermal growth factor receptor (EGFR) inhibitors and immune checkpoint inhibitors (ICIs), a prolonged survival time can be expected in some patients with cancers such as non-small cell lung cancer (NSCLC) $(7,8)$. Control of brain metastases may reduce the frequency of neurological symptoms and preserve quality of life.

A multicenter prospective observational study of gamma knife therapy for brain metastases that met the following criteria; 1) 10 or fewer in number, 2) maximal lesion size $<3 \mathrm{~cm}, 3)$ maximal lesion volume $<10 \mathrm{ml}$, and 4) total cumulative volume of $\leq 15 \mathrm{ml}$, demonstrated that gamma knife therapy for 5-10 brain metastases is not inferior to the treatment effect for 2-4 brain metastases (9). Thereafter, stereotactic radiosurgery (SRS) has been considered as the first line treatment even for multiple brain metastases.

In addition, technological developments have recently enabled us to utilize a single-isocenter multitarget (SIMT) SRS, which simultaneously irradiates multiple brain metastases at off-isocenter in linear accelerator (LINAC)based radiotherapy equipment (10). Similar to gamma-knife, $\mathrm{X}$-rays are expected to simultaneously irradiate multiple brain metastases with SRS. Therefore, LINAC-based SIMT SRS may be an option for the initial treatment of multiple brain metastases. Because SIMT SRS irradiates the target lesions off-center, even a slight positional error in the isocenter may result in larger errors of dose distribution to the target lesions. To the best of our knowledge, no previous studies have examined the effect of distance of each metastasis from the isocenter on local control. 
The purpose of this study was to examine whether the distance of each radiation target from the isocenter affects the local control of multiple brain metastases when treated with SIMT SRS with quantitative measurements in follow-up examinations using brain magnetic resonance imaging (MRI).

\section{Patients and Methods}

Patient selection. Twenty-two patients who received a first SIMT SRS for brain metastases derived from lung cancer at Shiga University of Medical Science Hospital between June 2016 and September 2018 were initially enrolled. Among the 22 patients, 4 patients in which the brain had been irradiated using gamma knife, $\mathrm{X}$ knife, or whole-brain irradiation before SIMT SRS were excluded. Moreover, 5 patients who did not undergo follow-up MRI examinations due to transfer or death immediately after SIMT SRS were also excluded. Therefore, 13 patients were evaluated. Of the 13 patients, 6 underwent SIMT SRS once. Among the remaining 7 cases, 4 and 3 cases underwent treatment two and three times, respectively, because of newly appeared metastases during the follow-up period. Among the 4 patients who underwent SIMT SRS twice, 1 patient died immediately after the second SRS due to lung cancer. The metastasis in which the second SRS was performed on was excluded from this study because no follow-up MRI was performed. Finally, 13 patients with 113 metastases were evaluated in this study (Table I). Locally recurrent nodules were re-irradiated with SIMT SRS for 3 cases. Four cases received additional whole brain irradiation due to re-growth of irradiated nodules and multiple new intracranial lesions. Systemic therapy was applied in all 13 cases. Tyrosine kinase inhibitors (TKIs) and ICIs were used in 5 and 5 cases, respectively. To measure subject condition, we used the Karnofsky performance status (KPS), Recursive Partitioning Analysis (RPA) (11), and Graded Prognostic Assessment (GPA) (12). This study was approved and monitored by the institutional ethics committee of Shiga University of Medical Science. The need to obtain informed patient consent was waived because of the retrospective nature of this research (Shiga University of Medical Science; R2018-143).

Radiation therapy. Image data for treatment planning was obtained using dedicated computed tomography (CT) (GE Lightspeed RT 16 system, GE Healthcare, Waukesha, WI, USA), with the head of the patient maintained in a fixed position using a stereotaxic head fixation mask (Brainlab, Munich, Germany). Within a couple of days before the date of the treatment planning CT, patients also underwent MRI with gadolinium enhancement and multiplanar reconstructed images in sagittal, coronal and trans-axial views were obtained based on a contrast-enhanced 3-dimensional T1-weighted image (CE-3D-T1WI). Treatment planning of SIMT SRS with dynamic conformal arc (DCA) was executed using dedicated treatment planning software (Multiple Brain Mets SRS, Brainlab) ver1.0.2 until December 28, 2017 and ver1.5.0 thereafter. CT images and CE-3D-T1WI were automatically fused using the software with minimal manual intervention by radiation oncologists. Sequentially, organs at risk such as brain stem, lens, eyeball, optic nerve, and hippocampus were highlighted with minimal required modification by radiation oncologists. For each of the brain metastasis, gross tumor volume (GTV) on CE-3DT1WI was determined with manual segmentation by radiation
Table I. Patient characteristics.

\begin{tabular}{|c|c|c|}
\hline & & $\%$ \\
\hline \multicolumn{3}{|l|}{ Age (yr) } \\
\hline Median \pm SD & $67 \pm 13$ & \\
\hline \multicolumn{3}{|l|}{ Gender } \\
\hline Female & 3 & 23 \\
\hline Male & 10 & 77 \\
\hline \multicolumn{3}{|c|}{ Karnofsky performance status } \\
\hline 90.100 & 7 & 54 \\
\hline 70,80 & 4 & 31 \\
\hline 50,60 & 1 & 8 \\
\hline 30,40 & 1 & 8 \\
\hline$\leq 20$ & 0 & 0 \\
\hline \multicolumn{3}{|c|}{ Recursive partioning analysis } \\
\hline Class 1 & 0 & 0 \\
\hline Class 2 & 11 & 85 \\
\hline Class 3 & 2 & 15 \\
\hline \multicolumn{3}{|l|}{ Diagnosis-specific GPA } \\
\hline $0-1.0$ & 6 & 46 \\
\hline $1.5-2.0$ & 4 & 31 \\
\hline $2.5-3.0$ & 3 & 23 \\
\hline $3.5-4.0$ & 0 & 0 \\
\hline \multicolumn{3}{|l|}{ Histological status } \\
\hline NSCLC & 11 & 85 \\
\hline Small cell & 2 & 15 \\
\hline \multicolumn{3}{|l|}{ Tyrosine kinase inhibitors } \\
\hline+ & 5 & 38 \\
\hline- & 8 & 62 \\
\hline \multicolumn{3}{|c|}{ Immune checkpoint inhibitors } \\
\hline+ & 5 & 38 \\
\hline- & 8 & 62 \\
\hline \multicolumn{3}{|l|}{ Acute advese effect } \\
\hline Facial nerve disorder & 1 (Grade 1$)$ & 8 \\
\hline Seizure & 1 (Grade 1$)$ & 8 \\
\hline None & 11 & 85 \\
\hline \multicolumn{3}{|c|}{ Number of new brain metastases } \\
\hline Median \pm SD & $6.0 \pm 3.5$ & \\
\hline Min-Max & $1-11$ & \\
\hline \multicolumn{3}{|l|}{$\operatorname{GTV}\left(\mathrm{cm}^{3}\right)$} \\
\hline Median \pm SD & $0.075 \pm 0.60$ & \\
\hline Min-Max & $0.011-4.1$ & \\
\hline \multicolumn{3}{|l|}{ Distribution of GTV } \\
\hline$\leq 1 \mathrm{~cm}^{3}$ & 109 & 96 \\
\hline$>1 \mathrm{~cm}^{3}$ & 4 & 4 \\
\hline \multicolumn{3}{|l|}{ Total PTV $\left(\mathrm{cm}^{3}\right)$} \\
\hline $\operatorname{median} \pm \mathrm{SD}$ & $2.0 \pm 2.7$ & \\
\hline Min-Max & $0.17-8.2$ & \\
\hline \multicolumn{3}{|c|}{$\begin{array}{l}\text { Distance between isocenter } \\
\text { and metastasis }(\mathrm{cm})\end{array}$} \\
\hline Median \pm SD & $4.9 \pm 1.9$ & \\
\hline Min-Max & $0-9.5$ & \\
\hline \multicolumn{3}{|l|}{ Necrosis } \\
\hline+ & 4 & 4 \\
\hline- & 109 & 96 \\
\hline
\end{tabular}

SD: Standard deviation; GPA: graded prognostic assessment; NSCLC: non-small lung cancer; Min: minimum; Max: maximum; GTV: gross tumor volume; PTV: planning target volume. 
oncologists. Clinical target volume (CTV) was defined as identical to the GTV. Planning target volume (PTV) - CTV margins were basically $1 \mathrm{~mm}$, with the exception of $2 \mathrm{~mm}$ for cases involving very small tumors, and $0.5 \mathrm{~mm}$ for a case with more than 10 tumors. We prescribed D99.5\% of PTV as 24 Gy in ver1.0.2 and D99.0\% of PTV as 24 Gy in ver1.5.0, except for one large metastasis, for which the prescription dose was modified to $20 \mathrm{~Gy}$ at the discretion of the radiation oncologist in charge. With the software, a DCA plan was automatically created to target multiple PTVs with a single isocenter. If some of the multiple targets were too far apart from one another, the software also automatically created a plan to divide the targets into two groups and irradiate each of them with respective single isocenters. The dose rate was 6 MV-SRS 1000 motor unit/min using Novalis Tx (Varian Medical Systems, Inc., Palo Alto, CA, USA). For each arc therapy, the bed location was modified slightly so that its tracking corresponded with a highly accurate patient monitoring system (ExacTrac System version6, BrainLab), with positional errors confined within $0.5 \mathrm{~mm}$ and 0.5 degrees.

When new brain metastases appeared in other regions during the follow-up period, additional SIMT SRS was performed. If the progression of brain metastasis was rapid, SIMT SRS was converted to whole-brain irradiation therapy at the discretion of the radiation oncologist. For local recurrent metastasis, up to one further SIMT SRS was allowed based on consensus between the radiation oncologists and attending physician.

Verification of dose distribution. Until December 28, 2017, after the treatment plan was transferred to a dedicated integrated treatment planning system (Eclipse version 13.6.23, Varian Medical System), a verification plan was developed by using the Acuros External Beam algorithm Version 13.6.23 (Acuros XB) in Delta ${ }^{4}$ (ScandiDos, Uppsala, Sweden) and examined with distance to agreement to check the positional accuracy of the multi-leaf collimator. In addition, a cylindrical phantom (MP Phantom, Toyo Medic Co., Ltd., Tokyo, Japan) dedicated to dose control containing more than 30 spaces inside for the placement of dosimeters was fixed in a head fixing shell (Brainlab) and CT image data were obtained using the same scanner as described above. Another verification plan was developed in the integrated treatment planning system with a cylindrical phantom. A PinPoint 3D Ion Chamber TN31016 Farmer (PTW, Freiburg, Germany) was installed as an ionization chamber in each of the inside spaces in the dedicated phantom. RAMTEC Smart (Toyo Medic Co., Ltd., Tokyo, Japan) was used as a potentiometer. Dose was measured with a $6 \mathrm{MV}$-SRS 1,000 monitor unit/min using Novalis Tx.

After December 29, 2017, the verification plan was developed directly in Delta4 with Multiple Brain Mets SRS ver1.5.0 to check the positional accuracy of the multi-leaf collimator in case of multiple targets. For cases with only one target, a verification plan for the cylindrical phantom was created using Multiple Brain Mets SRS and verified by actual measurement with the PinPoint 3D Ion Chamber TN31016 Farmer and the potentiometer RAMTEC Smart.

Quantitative assessment of size changes in metastases, and evaluation of survival, and adverse events during the follow-up period. Each patient underwent MRI with gadolinium enhancement to obtain CE-3D-T1WI every 1 to 4 months during the first year, and thereafter every 1 to 6 months. We established tumor shrinkage effect by referring to Response Evaluation Criteria in Solid Tumors
(RECIST) and Response Assessment in Neuro-Oncology Brain Metastases criteria (RANO-BM) because tumor sizes of almost all the brain metastases were non-measurable in RECIST and RANO$\mathrm{BM}$. The maximum value of the longest diameters (LDs) in each of the sagittal, coronal and trans-axial planes measured manually by a radiation oncologist on CE-3D-T1WI was defined as the LD of the metastasis. Tumor reduction rate (TRR) was calculated as post-SRS $\mathrm{LD}$ at the respective time-point in the follow up period subtracted from the pre-SRS LD divided by the pre-SRS LD. Tumor shrinkage effects were classified into four grades according to their TRR at the last MRI examination date or the MRI examination date just before reirradiation, as follows; almost disappeared: $\mathrm{TRR}=1$, decreased: $0.3 \leq \mathrm{TRR}<1$, stable: $-0.2<\mathrm{TRR}<0.3$ and increased: TRR $\leq-0.2$. Exceptionally, when TRR got -0.2 or less at a time point during the follow-up period, tumor shrinkage effect was determined as increased at that time regardless of the subsequent TRR. Brain metastases classified as almost disappeared, decreased, and stable were defined as local control, and those increased as local failure. The distance between metastases and the isocenter was calculated from the respective tumor central coordinates using Eclipse. Brain metastases were classified into three other groups by trisecting the maximal distance of individual metastases from the isocenter as follows; near group with a distance of $<3.2 \mathrm{~cm}$, middle group with a distance of $\geq 3.2 \mathrm{~cm}$ and $<6.4 \mathrm{~cm}$, and far group with a distance of $\geq 6.4 \mathrm{~cm}$. The distribution of TRRs was compared among the 3 groups to investigate the effect of distance between the metastases and the isocenter on local control. Furthermore, two radiological oncologists assessed through consensus whether each metastasis became necrotized at the time of MRI examination by reviewing all the trans-axial slice images.

Overall survival was defined as the duration from the first SIMT SRS to the last follow-up point before December 31, 2020. Major adverse events were assessed based on the common terminology criteria for Adverse Event 5.0 (13).

Statistics analysis. $p$-Values of $<0.05$ were considered to indicate statistical significance. EZR version 1.38 (Saitama Medical Center, Jichi Medical University, Saitama, Japan) was used for statistical analysis (14). Survival and local control rates were determined using the Kaplan-Meier method. Local control rates were compared among the 3 groups using Log-rank test. Distribution in tumor shrinkage effects was compared among the 3 groups using the Kruskal-Wallis test.

\section{Results}

Overall survival. The follow-up period was $17 \pm 8.8$ months. Overall survival of the 13 patients who underwent SIMT SRS at 6, 12, and 18 months were 92\%, 69\%, and $31 \%$, respectively, with a median survival time of 17 months (Figure 1). All deaths were due to primary illness and no neurological deaths were observed.

Tumor size distribution and local control. The number of new brain metastases was $6.0 \pm 3.5$ (1-11). GTV was $0.075 \pm 0.60 \mathrm{~cm}^{3}\left(0.011-4.1 \mathrm{~cm}^{3}\right)$. Brain metastases with GTV of $\leq 1 \mathrm{~cm}^{3}$ accounted for the majority (96\%) of the total metastases and total PTV per SIMT SRS was 2.0 \pm 2.7 


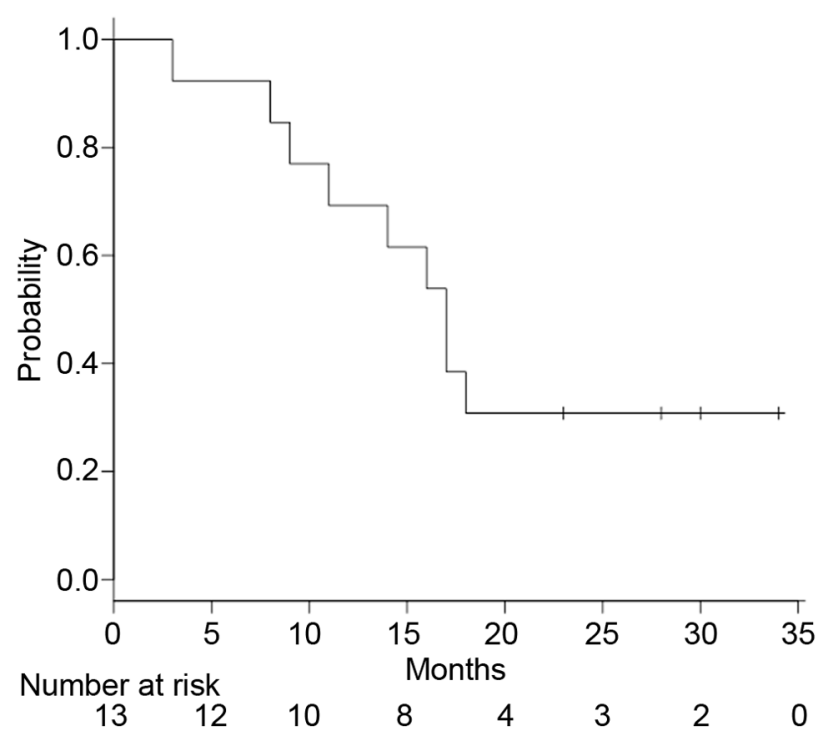

Figure 1. Kaplan-Meier curve demonstrating overall survival of total study population.

$\mathrm{cm}^{3}\left(0.17-8.2 \mathrm{~cm}^{3}\right)$ (Table I). The local control rates at 6 , 12 , and 18 months were $88 \%, 88 \%$, and $88 \%$, respectively (Figure 2A). In terms of the reduction rates, $63.7 \%, 11.5 \%$, $12.4 \%$, and $12.4 \%$ of the total brain metastases were classified into almost disappeared, decreased, stable, and increased, respectively (Figure 3A).

Association of the distance of metastases from the isocenter with tumor shrinkage effect. The distance between brain metastases and the isocenter was $4.9 \pm 1.9 \mathrm{~cm}(0-9.5 \mathrm{~cm})$ (Table I). Overall, 23, 71, and 19 metastases were classified into the near, middle, and far groups, respectively.

Local control in these 3 groups did not show significant difference in the evaluation using the Log Rank test (Figure 2B). Distribution in the effect on tumor shrinkage did not show any significant difference between the 3 groups, as almost disappeared, decreased, stable, and increased grades were $69.6 \%, 17.4 \%, 4.3 \%$, and $8.7 \%$ in the near group, $60.6 \%, 11.3 \%, 14.1 \%$, and $14.1 \%$ in the middle group, and $68.4 \%, 5.3 \%, 15.8 \%$, and $10.5 \%$ in the far group (Figure 3B and $\mathrm{C}$ ).

Adverse events. Facial nerve disorder grade 1 in 1 case and seizure grade 1 in another case were observed (Table I). No acute adverse events for headache, nausea, somnolence, amnesia, or ataxia were observed. Four brain necroses were found in 3 patients (Table I). Among them, one patient was treated with single SRS and the other two were treated with radiation therapy as post-treatment.

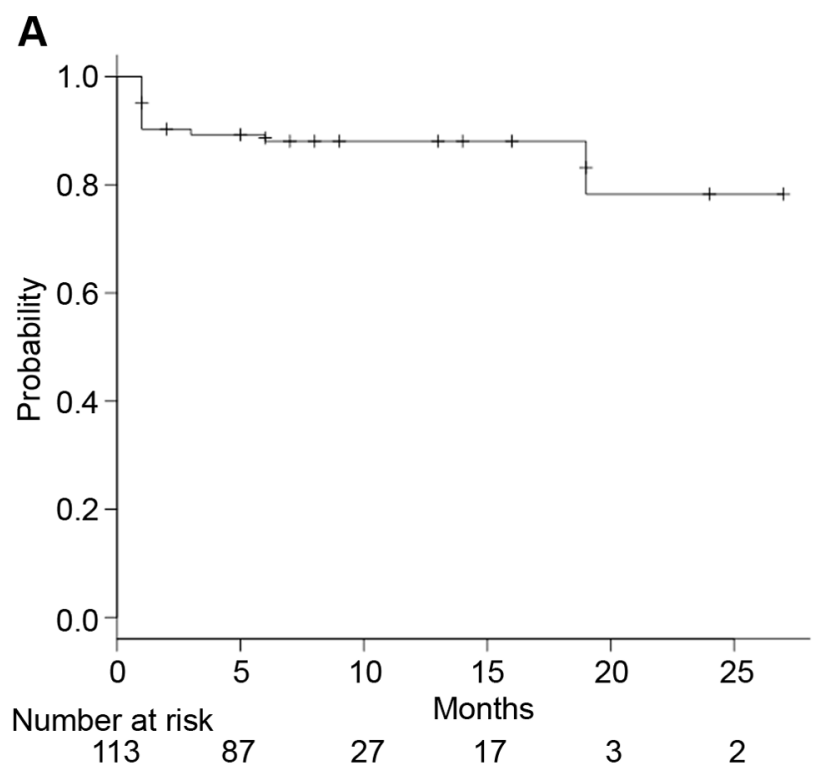

B

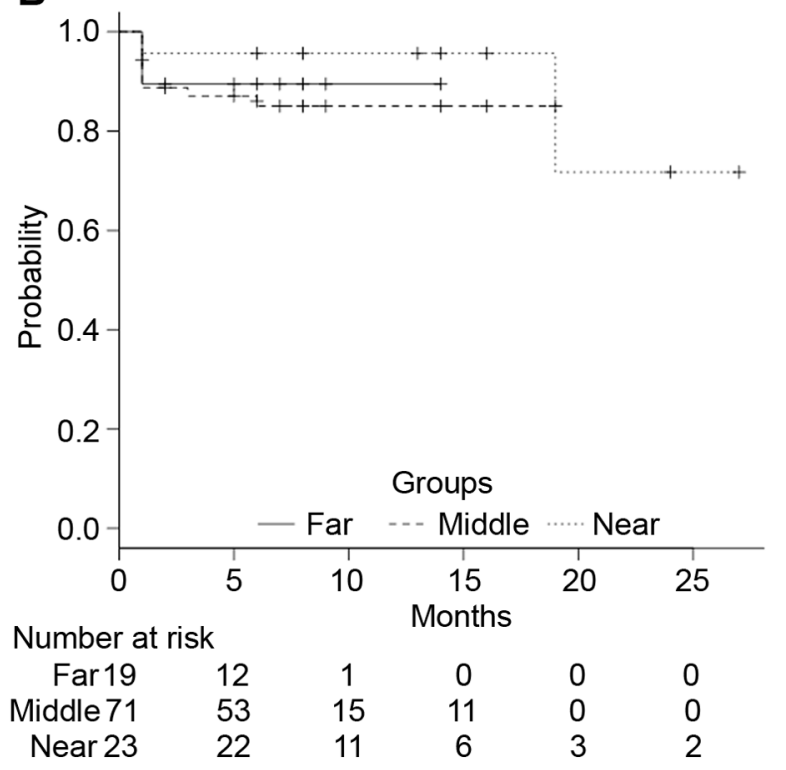

Figure 2. Kaplan-Meier curve demonstrating local control of brain metastasis for total brain metastases (A) and for each of the three groups classified by the distance between metastases and the isocenter (B). $p=0.69$.

\section{Discussion}

In this study, we quantified the size change in individual brain metastases during follow-up after SIMT SRS on CE-3D-T1WI and found that: 1) although distance between the isocenter and individual metastases varied from 0 to $9.5 \mathrm{~cm}$, total local control rates were good at $88 \%$ and $88 \%$ at 6 and 12 months; 2) local control rates of individual metastases were maintained even with increasing distances from the isocenter. 
In this study, local control rates by SIMT SRS for brain metastases of lung cancers were excellent at 1 year. Among various treatments such as surgical resection, chemotherapy, and irradiation, SRS may be regarded as a safe and effective treatment option for multiple brain metastases (15). In a previous report on initial experiences with SIMT SRS in 31 patients with a median clinical follow-up of 11 months, 1year survival was $68 \%$ and local control was $89 \%$ (16), which is comparable to the results in this study. LINACbased SRS, which is widely adopted in many hospitals and institutions, generally irradiates only a single brain metastasis as a target, and it requires long treatment times for cases with multiple brain metastases, resulting in patient burden. Therefore, the favorable results demonstrated in this study for cases with brain metastases with simultaneous SIMT SRS are clinically important.

Local control rates in individual metastases were maintained irrespective of the distance between metastases and the isocenter, although this distance varied from 0 to 9.5 $\mathrm{cm}$. Tumor shrinkage effects were also similar in the 3 groups of brain metastases categorized based on the distance of the lesion from isocenter. The rate of category of almost disappeared was $69.6 \%, 60.6 \%$, and $68.4 \%$, in the near, middle, and far groups, respectively. Correction of rotational error has been reported to be important in the treatment of multiple brain metastases using volumetric modulated arc therapy (VMAT) with a single isocenter $(17,18)$. Selvan et $a l$. reported that in SIMT SRS for multiple brain metastases using VMAT, the distance from the target lesion to the isocenter was associated with rotational error and strongly influenced the target coverage. The smaller the target volume, the more likely the rotational error was compared to the larger target volume (18). In this study, we carefully irradiated brain metastases with accurate locational adjustment using ExacTrac, with positional errors confined within $0.5 \mathrm{~mm}$ and 0.5 degrees. We believe that decline of target coverage of metastases far from the isocenter can be avoided by accurate patient positioning.

It should be noted that the local control rates of this study were comparable to those of previous studies using gamma knife even though the majority of metastases were small (median GTV: $0.075 \mathrm{~cm}^{3}$ ). In a previous study, Serizawa et al. categorized brain metastases into tiny $\left(\leq 1.0 \mathrm{~cm}^{3}\right)$ and small $\left(>1.0 \mathrm{~cm}^{3}\right.$ to $\left.\leq 4.0 \mathrm{~cm}^{3}\right)$ groups and treated them with gamma knife. They showed 1-year tumor control rates of 98.4\% and $92.3 \%$ for tiny and small lesions, respectively (19). Our study indicates the potential of SIMT SRS using DCA to treat small brain metastases, with comparable local control to gamma knife.

We performed SIMT SRS on 1-11 multiple brain metastasis and showed comparable overall survival; the median overall survival for all the cases was 17 months. In two previous studies, SRS for 1-3 brain metastases resulted
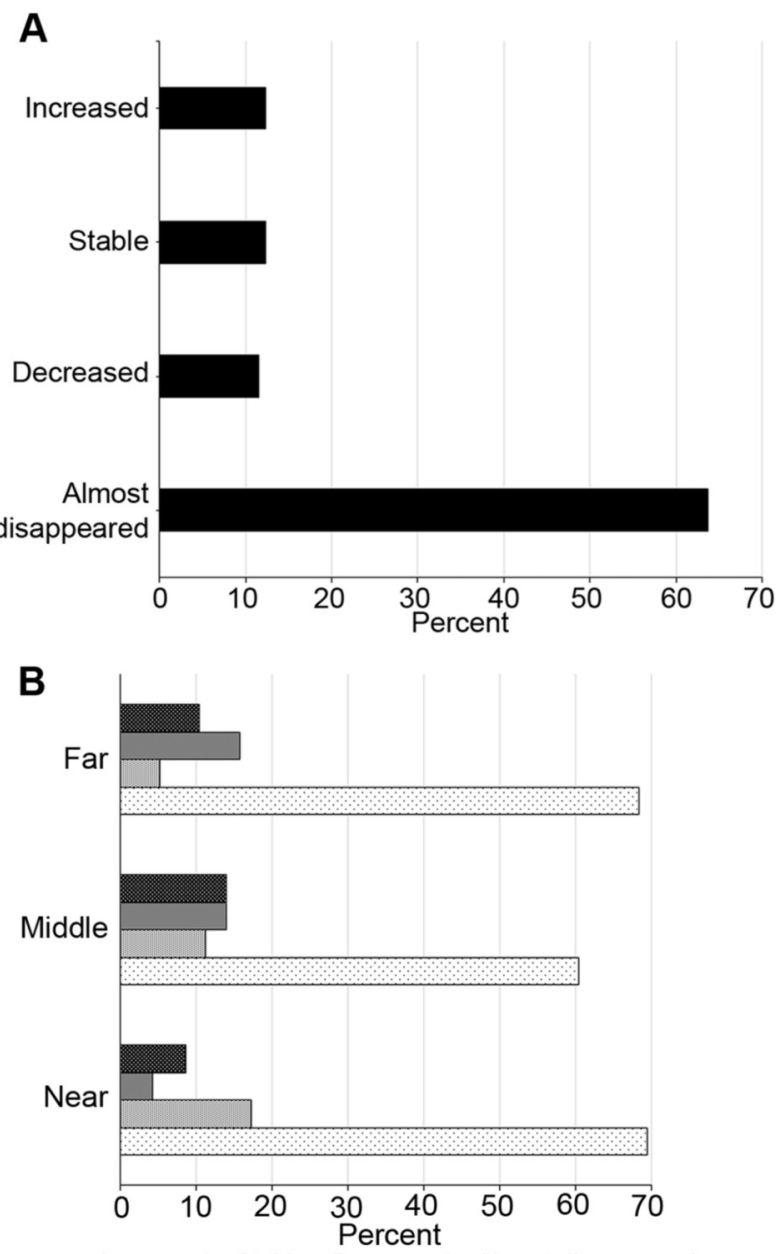

\. Increased $₫$ Stable $\square$ Decreased $₫$ Almost disappeared

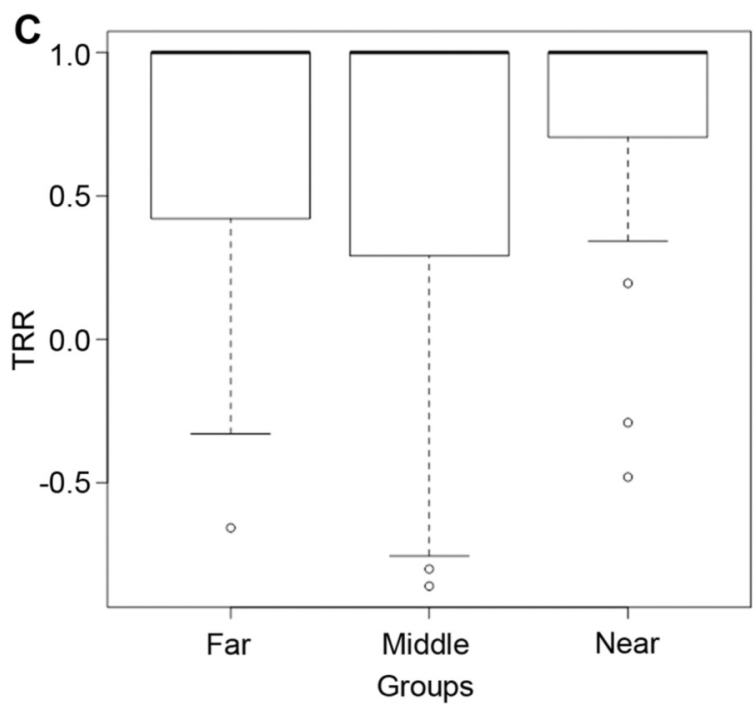

Figure 3. Bar graphs demonstrating the distribution in the tumor shrinkage effect for total brain metastases (A), for each of the three groups classified by the distance between metastases and the isocenter $(B)$, and in the tumor reduction rate (TRR) for each of the three groups classified by the distance between metastases and the isocenter $(C) . p=0.58$. 
in median survival times of 15.2 and 10.4 months, respectively $(5,20)$. Vesagas et al. reported that median survival of 8.4 months was obtained using SRS with a gamma knife on brain metastases (21). In a recent research, Palmer et al. reported that SIMT radiotherapy in 1 to 5 fractions for brain metastases using VMAT resulted in a median survival of 13.2 months (22). Although a limited number of cases were included in this study, SIMT SRS using DCA may be comparable to SRS with X-ray and gamma knife. Since molecular targeted drugs and ICIs were introduced into routine clinical settings, systemic therapy has been improved, with SRS for brain metastases is increasingly administered sequentially. In another recent study, Yomo et al. demonstrated a better survival in patients who underwent gamma knife for brain metastases with an EGFR-TKI as compared to those without (23). Magnuson et al. reported that sequential use of SRS and TKI could prolong median survival time in EGFR-mutated NSCLC patients with brain metastases (24). The population of our study was patients with NSCLC $(85 \%)$, and $38 \%$ of the study population underwent treatment with TKIs, which might have had favorable effect. The relatively favorable overall survival seen in this study may be attributable to the inclusion criteria used, that is, only patients who could undergo followed-up MRI examination at more than 1 month after SIMT SRS were assessed. In addition, $85 \%$ of patients had KPS $>70$ and $77 \%$ had diagnosis-specific GPA $\leq 2.0$, which may have contributed to the overall survival. Nonetheless, SIMT SRS could provide comparable overall survival to conventional treatment strategies for multiple brain metastases and a multicenter trial is warranted to verify this possibility.

Severe adverse events were not confirmed during SIMT SRS, although acute adverse events up to grade 1 were observed. Death due to lung cancer was confirmed within the survey period, and no neurological deaths were observed. Among 113 total brain metastases in 13 patients, 4 necroses, which composed of 2 in near and 2 in middle group, were detected in 3 patients on MRI examinations. Therefore, any association of the distance from the isocenter with tumor necrosis could not be clearly demonstrated. Because we could not investigate brain necrosis in detail by using biopsy or $11 \mathrm{C}$-methionine-positron emission tomography, we may have overlooked some brain metastases, which were actually necrotized. Precise evaluation is required to demonstrate this relationship in a large number of future cases.

In conclusion, effective local control of small multiple brain metastases was achieved by SIMT SRS using DCA irrespective of the distance of metastases from the isocenter.

\section{Conflicts of Interest}

The Authors have no conflicts of interest to disclose regarding this study.

\section{Authors' Contributions}

KA: methodology, data curation, statistics, writing-original draft, review, editing. YN: data curation, statistics, review, editing. $\mathrm{KN}$ : data curation, review, editing. TT: data curation, statistics. NK: data curation. YK: review, editing. NH: data curation. YT: data curation. YW: supervision.

\section{References}

1 Bertolini F, Spallanzani A, Fontana A, Depenni R and Luppi G: Brain metastases: an overview. CNS Oncol 4(1): 37-46, 2015. PMID: 25586424. DOI: $10.2217 / \mathrm{cns} .14 .51$

2 Schouten LJ, Rutten J, Huveneers HA and Twijnstra A: Incidence of brain metastases in a cohort of patients with carcinoma of the breast, colon, kidney, and lung and melanoma. Cancer 94(10): 2698-2705, 2002. PMID: 12173339. DOI: $10.1002 /$ cncr. 10541

3 Nieder C, Spanne O, Mehta MP, Grosu AL and Geinitz H: Presentation, patterns of care, and survival in patients with brain metastases: what has changed in the last 20 years? Cancer 117(11): 2505-2512, 2011. PMID: 24048799. DOI: 10.1002/cncr. 25707

4 Soffietti R, Rudā R and Mutani R: Management of brain metastases. J Neurol 249(10): 1357-1369, 2002. PMID: 12382150. DOI: $10.1007 / \mathrm{s} 00415-002-0870-6$

5 Chang EL, Wefel JS, Hess KR, Allen PK, Lang FF, Kornguth DG, Arbuckle RB, Swint JM, Shiu AS, Maor MH and Meyers CA: Neurocognition in patients with brain metastases treated with radiosurgery or radiosurgery plus whole-brain irradiation: a randomised controlled trial. Lancet Oncol 10(11): 1037-1044, 2009. PMID: 19801201. DOI: 10.1016/S1470-2045(09)70263-3

6 Brown PD, Ballman KV, Cerhan JH, Anderson SK, Carrero XW, Whitton AC, Greenspoon J, Parney IF, Laack NNI, Ashman JB, Bahary JP, Hadjipanayis CG, Urbanic JJ, Barker FG 2nd, Farace E, Khuntia D, Giannini C, Buckner JC, Galanis E and Roberge D: Postoperative stereotactic radiosurgery compared with whole brain radiotherapy for resected metastatic brain disease (NCCTG N107C/CEC.3): a multicentre, randomised, controlled, phase 3 trial. Lancet Oncol 18(8): 1049-1060, 2017. PMID: 28687377. DOI: $10.1016 / \mathrm{S} 1470-2045(17) 30441-2$

7 Yang JC, Wu YL, Schuler M, Sebastian M, Popat S, Yamamoto N, Zhou C, Hu CP, O’Byrne K, Feng J, Lu S, Huang Y, Geater SL, Lee KY, Tsai CM, Gorbunova V, Hirsh V, Bennouna J, Orlov S, Mok T, Boyer M, Su WC, Lee KH, Kato T, Massey D, Shahidi M, Zazulina V and Sequist LV: Afatinib versus cisplatinbased chemotherapy for EGFR mutation-positive lung adenocarcinoma (LUX-Lung 3 and LUX-Lung 6): analysis of overall survival data from two randomised, phase 3 trials. Lancet Oncol 16(2): 141-151, 2015. PMID: 25589191. DOI: 10.1016/S1470-2045(14)71173-8

8 Reck M, Rodríguez-Abreu D, Robinson AG, Hui R, Csőszi T, Fülöp A, Gottfried M, Peled N, Tafreshi A, Cuffe S, O'Brien M, Rao S, Hotta K, Leiby MA, Lubiniecki GM, Shentu Y, Rangwala R, Brahmer JR and KEYNOTE-024 Investigators: Pembrolizumab versus chemotherapy for PD-L1-positive nonsmall-cell lung cancer. N Engl J Med 375(19): 1823-1833, 2016. PMID: 27718847. DOI: 10.1056/NEJMoa1606774

9 Yamamoto M, Serizawa T, Shuto T, Akabane A, Higuchi Y, Kawagishi J, Yamanaka K, Sato Y, Jokura H, Yomo S, Nagano 
O, Kenai H, Moriki A, Suzuki S, Kida Y, Iwai Y, Hayashi M, Onishi H, Gondo M, Sato M, Akimitsu T, Kubo K, Kikuchi Y, Shibasaki T, Goto T, Takanashi M, Mori Y, Takakura K, Saeki N, Kunieda E, Aoyama H, Momoshima S and Tsuchiya K: Stereotactic radiosurgery for patients with multiple brain metastases (JLGK0901): a multi-institutional prospective observational study. Lancet Oncol 15(4): 387-395, 2014. PMID: 24621620. DOI: 10.1016/S1470-2045(14)70061-0

10 Gevaert T, Steenbeke F, Pellegri L, Engels B, Christian N, Hoornaert MT, Verellen D, Mitine $\mathrm{C}$ and De Ridder M: Evaluation of a dedicated brain metastases treatment planning optimization for radiosurgery: a new treatment paradigm? Radiat Oncol 11: 13, 2016. PMID: 26831367. DOI: 10.1186/s13014016-0593-y

11 Gaspar L, Scott C, Rotman M, Asbell S, Phillips T, Wasserman T, McKenna WG and Byhardt R: Recursive partitioning analysis (RPA) of prognostic factors in three Radiation Therapy Oncology Group (RTOG) brain metastases trials. Int J Radiat Oncol Biol Phys 37(4): 745-751, 1997. PMID: 9128946. DOI: 10.1016/s0360-3016(96)00619-0

12 Sperduto PW, Kased N, Roberge D, Xu Z, Shanley R, Luo X, Sneed PK, Chao ST, Weil RJ, Suh J, Bhatt A, Jensen AW, Brown PD, Shih HA, Kirkpatrick J, Gaspar LE, Fiveash JB, Chiang V, Knisely JP, Sperduto CM, Lin N and Mehta M: Summary report on the graded prognostic assessment: an accurate and facile diagnosis-specific tool to estimate survival for patients with brain metastases. J Clin Oncol 30(4): 419-425, 2012. PMID: 22203767. DOI: 10.1200/JCO.2011.38.0527

13 Common Terminology Criteria for Adverse Events (CTCAE) Version 5.0. Available at: https://ctep.cancer.gov/protocol development/electronic_applications/docs/CTCAE_v5_Quick_Ref erence_5x7.pdf\#search $=\% 27$ the+common+terminology+criteria + fo r+Adverse+Event+5.0\%27 [Last accessed on March 21, 2021]

14 Kanda Y: Investigation of the freely available easy-to-use software 'EZR' for medical statistics. Bone Marrow Transplant 48(3): 452-458, 2013. PMID: 23208313. DOI: 10.1038/bmt. 2012.244

15 Kraft J, Zindler J, Minniti G, Guckenberger M and Andratschke $\mathrm{N}$ : Stereotactic radiosurgery for multiple brain metastases. Curr Treat Options Neurol 21(2): 6, 2019. PMID: 30758726. DOI: 10.1007/s11940-019-0548-3

16 Minniti G, Capone L, Alongi F, Figlia V, Nardiello B, El Gawhary R, Scaringi C, Bianciardi F, Tolu B, Gentile P and Paolini S: Initial experience with single-isocenter radiosurgery to target multiple brain metastases using an automated treatment planning software: clinical outcomes and optimal target volume margins strategy. Adv Radiat Oncol 5(5): 856-864, 2020. PMID: 33083647. DOI: 10.1016/j.adro.2020.06.008

17 Roper J, Chanyavanich V, Betzel G, Switchenko J and Dhabaan A: Single-isocenter multiple-target stereotactic radiosurgery: risk of compromised coverage. Int J Radiat Oncol Biol Phys 93(3): 540546, 2015. PMID: 26460996. DOI: 10.1016/j.ijrobp.2015.07.2262
18 Selvan KT, Padma G, Revathy MK, Nambi Raj NA, Senthilnathan K and Babu PR: Dosimetric effect of rotational setup errors in single-isocenter volumetric-modulated arc therapy of multiple brain metastases. J Med Phys 44(2): 84-90, 2019. PMID: 31359925. DOI: 10.4103/jmp.JMP_103_18

19 Serizawa T, Hirai T, Nagano O, Higuchi Y, Matsuda S, Ono J and Saeki N: Gamma knife surgery for 1-10 brain metastases without prophylactic whole-brain radiation therapy: analysis of cases meeting the Japanese prospective multi-institute study (JLGK0901) inclusion criteria. J Neurooncol 98(2): 163-167, 2010. PMID: 20411300. DOI: 10.1007/s11060-010-0169-x

20 Brown PD, Jaeckle K, Ballman KV, Farace E, Cerhan JH, Anderson SK, Carrero XW, Barker FG 2nd, Deming R, Burri SH, Ménard C, Chung C, Stieber VW, Pollock BE, Galanis E, Buckner JC and Asher AL: Effect of radiosurgery alone vs radiosurgery with whole brain radiation therapy on cognitive function in patients with 1 to 3 brain metastases: a randomized clinical trial. JAMA 316(4): 401-409, 2016. PMID: 27458945. DOI: $10.1001 /$ jama.2016.9839

21 Vesagas TS, Aguilar JA, Mercado ER and Mariano MM: Gamma knife radiosurgery and brain metastases: local control, survival, and quality of life. J Neurosurg 97(5 Suppl): 507-510, 2002. PMID: 12507086. DOI: 10.3171/jns.2002.97.supplement

22 Palmer JD, Sebastian NT, Chu J, DiCostanzo D, Bell EH, Grecula J, Arnett A, Blakaj DM, McGregor J, Elder JB, Lu L, Zoller W, Addington M, Lonser R, Chakravarti A, Brown PD and Raval R: Single-isocenter multitarget stereotactic radiosurgery is safe and effective in the treatment of multiple brain metastases. Adv Radiat Oncol 5(1): 70-76, 2019. PMID: 32051892. DOI: $10.1016 /$ j.adro.2019.08.013

23 Yomo S, Serizawa T, Yamamoto M, Higuchi Y, Sato Y, Shuto T, Akabane A, Jokura H, Kawagishi J and Aoyama H: The impact of EGFR-TKI use on clinical outcomes of lung adenocarcinoma patients with brain metastases after Gamma Knife radiosurgery: a propensity score-matched analysis based on extended JLGK0901 dataset (JLGK0901-EGFR-TKI). J Neurooncol 145(1): 151-157, 2019. PMID: 31487030. DOI: 10.1007/s11060-019-03282-0

24 Magnuson WJ, Lester-Coll NH, Wu AJ, Yang TJ, Lockney NA, Gerber NK, Beal K, Amini A, Patil T, Kavanagh BD, Camidge DR, Braunstein SE, Boreta LC, Balasubramanian SK, Ahluwalia MS, Rana NG, Attia A, Gettinger SN, Contessa JN, Yu JB and Chiang VL: Management of brain metastases in tyrosine kinase inhibitor-naïve epidermal growth factor receptor-mutant nonsmall-cell lung cancer: a retrospective multi-institutional analysis. J Clin Oncol 35(10): 1070-1077, 2017. PMID: 28113019. DOI: $10.1200 / J C O .2016 .69 .7144$

Received March 31, 2021

Revised April 15, 2021

Accepted April 16, 2021 